\title{
Reducing numerical diffusion in magnetospheric simulations
}

\author{
Gábor Tóth, ${ }^{1}$ Xing Meng, ${ }^{1}$ Tamas I. Gombosi, ${ }^{1}$ and Aaron J. Ridley ${ }^{1}$ \\ Received 10 December 2010; revised 8 March 2011; accepted 14 April 2011; published 13 July 2011.
}

[1] Physics-based global magnetosphere modeling requires large computational resources. It is still impractical to resolve the computational domain to the point where numerical errors become negligible. One possible way of reducing numerical diffusion is the "Boris correction": the semirelativistic magnetohydrodynamics equations are solved with an artificially reduced speed of light. Here we introduce a new alternative approach, an Implicit Scheme with Limited Numerical Dissipation (ISLND). The fully implicit time stepping provides stability, and the wave speeds are limited in the dissipative numerical fluxes only. This limiting only affects the numerical scheme, and it does not modify the equations being solved. This approach can be employed for most total variation diminishing schemes. The differences between the Boris and ISLND schemes are demonstrated in simple numerical tests. We also perform several simulations for two magnetic storms using the global magnetosphere, the ionosphere electrodynamics, and the inner magnetosphere models of the Space Weather Modeling Framework, and we compare the Boris scheme with the limited numerical dissipation method and also with the unmodified base scheme at various grid resolutions. We find that for these particular simulations the Boris scheme and the ISLND scheme produce comparable results, both being significantly less diffusive than the unmodified scheme.

Citation: Tóth, G., X. Meng, T. I. Gombosi, and A. J. Ridley (2011), Reducing numerical diffusion in magnetospheric simulations, J. Geophys. Res., 116, A07211, doi:10.1029/2010JA016370.

\section{Introduction}

[2] Physics-based modeling of the global magnetosphere around the Earth and other magnetized planets has been pursued for decades. Most models are based on a fluid description of the plasma and solve for some form of the magnetohydrodynamic (MHD) equations [e.g., Powell et al., 1999; Lyon et al., 2004; Raeder et al., 2001; Palmroth et al., 2003; Tanaka, 1995]. While MHD is clearly a simplification of the physics of the low density plasma of the magnetosphere, it gives surprisingly good predictions for the position of the bow shock or the magnetopause. On the other hand the MHD model is not really accurate in the inner magnetosphere where high energy particles are trapped on closed magnetic field lines. The inner boundary of the magnetosphere is typically the ionosphere of the planet (or moon), which is another complicated system that cannot be well represented by the MHD equations. To achieve a better description of the whole magnetospheric system, one has to couple the global magnetosphere (GM) model with an ionospheric electrodynamics (IE) model and preferably with an inner magnetosphere (IM) model as well [De Zeeuw et al., 2004; Toffoletto et al., 2004].

\footnotetext{
${ }^{1}$ Department of Atmospheric Oceanic and Space Science, University of Michigan, Ann Arbor, Michigan, USA.

Copyright 2011 by the American Geophysical Union. 0148-0227/11/2010JA016370
}

[3] The accuracy of the magnetosphere model is not limited by the various approximations in the physics only. The equations have to be solved numerically, and even on today's supercomputers, it is not feasible to obtain a "grid converged" solution [Ridley et al., 2010], i.e., a numerical solution in which the effect of the numerical errors is negligible for the quantities of interest (e.g., the magnetic field and plasma parameters along a given satellite trajectory, or the Dst index).

[4] In this paper, we concentrate on the numerical errors of the GM model that solves the time-dependent MHD equations in a large three-dimensional (3-D) domain. Numerical errors can be dispersive leading to spurious oscillations, or dissipative leading to an overly smooth solution. The shock-capturing schemes used in modern MHD codes tend to suppress spurious oscillations quite well, so most of the numerical error occurs in form of numerical diffusion. In terms of the coupled GM-IM-IE system, the numerical diffusion in GM will mostly affect the accuracy and amplitude of the field-aligned currents used in the GM-IE coupling, and also the sharpness and amplitude of the pressure gradients driven by the IM-GM coupling. The numerical diffusion also limits how effectively the IE drift velocities influence the GM solution further away from the inner boundary.

[5] We will describe the form of numerical diffusion in section 2 and explore possible ways to reduce the diffusion and the associated numerical error. In particular we will describe a new approach, an Implicit Scheme with Limited Numerical Dissipation (ISLND) that reduces the numerical 
diffusion without modifying the original equations. The properties of the various numerical schemes are compared on simple tests in section 3 , while section 4 presents magnetospheric simulations and also provides comparison with data. We simulate two storms to make sure that our results are not specific to a single storm. Finally we draw conclusions in section 5 .

\section{How to Reduce Numerical Diffusion}

[6] We are considering total variation diminishing (TVD) type shock capturing schemes [van Leer, 1979; Harten, 1983], in particular the Lax-Friedrichs (LF) or Rusanov [Rusanov, 1970], the Harten-Lax-van Leer (HLL) [Harten et al., 1983] and Roe schemes [Roe, 1981]. In these finite volume schemes the numerical flux is calculated at the cell interfaces and it depends on the (interpolated) right and left states $U^{R}$ and $U^{L}$. The state vector $U$ can consist of the primitive variables (density, pressure, three components of velocity, and three components of the magnetic field), or conservative variables (mass density, energy density, momentum density, and magnetic field). The flux functions of the three schemes are

$$
\begin{gathered}
F^{\mathrm{LF}}=\frac{F\left(U^{R}\right)+F\left(U^{L}\right)}{2}-\frac{1}{2}\left|\lambda_{\max }\right|\left(U^{R}-U^{L}\right) \\
F^{\mathrm{HLL}}=\frac{\lambda_{R} F\left(U^{L}\right)-\lambda_{L} F\left(U^{R}\right)}{\lambda_{R}-\lambda_{L}}+\frac{\lambda_{R} \lambda_{L}}{\lambda_{R}-\lambda_{L}}\left(U^{R}-U^{L}\right) \\
F^{\mathrm{Roe}}=\frac{F\left(U^{R}\right)+F\left(U^{L}\right)}{2}-\frac{1}{2} \sum_{k} l_{k}\left|\lambda_{k}\right| r_{k} \cdot\left(U^{R}-U^{L}\right)
\end{gathered}
$$

where $F$ is the physical flux function. In the Roe flux, $\lambda_{k}$ is the $k$ th eigenvalue (characteristic wave speed), and $l_{k}$ and $r_{k}$ are the left and right eigenvectors of $\partial F / \partial U$. In the LaxFriedrichs flux, $\lambda_{\max }=\max \left(\left|\lambda_{k}\right|\right)$, while in the HLL flux, $\lambda_{R}=$ $\max \left(0, \lambda_{k}\right)$ and $\lambda_{L}=\min \left(0, \lambda_{k}\right)$. The wave speeds are calculated for the left and right states and/or some averaged state. The details of these schemes are described in the references, and are not important for the following discussion.

[7] For all three schemes the flux functions consist of two terms. The first term contains the physical flux as a (weighted) average of $F\left(U^{R}\right)$ and $F\left(U^{L}\right)$. The second term is a correction term that is proportional to $\left(U^{R}-U^{L}\right)$ and some combination of the absolute values of $\lambda_{k}$. This correction term makes the schemes stable and oscillation free. For the Roe scheme the correction term selects the upwind flux for each characteristic wave. For the LF and HLL schemes the correction term acts as an upwinding correction for the fastest characteristic waves only. For slower waves, however, the correction term acts as a dissipative numerical flux, and it can be written as

$$
F_{\text {diss }}(U) \propto|\lambda|\left(U^{R}-U^{L}\right)
$$

In general $\lambda$ is an upper estimate on the characteristic wave speed associated with the jump between the two states. In the HLL scheme $\lambda$ is the harmonic average of the fastest right and left going wave speeds $\left(\lambda_{R}\right.$ and $\left.-\lambda_{L}\right)$, while in the LF scheme $\lambda$ is the largest of all wave speeds.
[8] In general, the Roe scheme is the least dissipative and the LF scheme is the most dissipative among the three schemes. The HLL scheme is in between. When the wave speeds are symmetric, the HLL scheme reduces to the LF scheme, since $\lambda_{R}=-\lambda_{L}=\lambda_{\max }$. This happens when the bulk velocity of the plasma is zero (or very small). The other extreme is a superfast (faster than the fast magnetosonic speed) flow when all the characteristic waves move in the same direction, for example to the right so that $\lambda_{L}=0$. In this case the HLL scheme becomes purely upwind and $F^{\mathrm{HLL}}=$ $F\left(U^{L}\right)$. In this paper, we focus on the numerical diffusion of the magnetic field perturbations in the closed field line region of the magnetosphere, where the flow speeds are small, and $\lambda$ is approximately the fast magnetosonic speed in equation (4).

[9] Away from discontinuities the $U^{R}-U^{L}$ difference can be approximated with a Taylor series expansion of the analytic $U$ around the face center located at $x_{i+1 / 2}$ between cells $i$ and $i+1$. For the spatially first-order scheme (or whenever the higher-order scheme reduces to first order) the left and right states are simply the same as the left and right cell center values:

$$
\begin{gathered}
U^{L}=U_{i}=U_{i+1 / 2}-\frac{\Delta x}{2} \frac{\partial U}{\partial x}+O\left(\Delta x^{2}\right) \\
U^{R}=U_{i+1}=U_{i+1 / 2}+\frac{\Delta x}{2} \frac{\partial U}{\partial x}+O\left(\Delta x^{2}\right)
\end{gathered}
$$

where $\Delta x$ is the grid cell size. Subtracting the two equations from each other, the difference is

$$
U^{R}-U^{L}=\Delta x \frac{\partial U}{\partial x}+O\left(\Delta x^{2}\right)
$$

Second-order TVD schemes use limited slopes to obtain $U^{R}$ and $U^{L}$ :

$$
\begin{gathered}
U^{L}=U_{i}+\frac{1}{2} \bar{\Delta} U_{i} \\
U^{R}=U_{i+1}-\frac{1}{2} \bar{\Delta} U_{i+1}
\end{gathered}
$$

The limited slope can be defined as [van Leer, 1979]

$$
\bar{\Delta} U_{i}=\operatorname{minmod}\left[\beta\left(U_{i+1}-U_{i}\right), \beta\left(U_{i}-U_{i-1}\right),\left(U_{i+1}-U_{i-1}\right) / 2\right]
$$

where the minmod function returns zero if any of the arguments have different signs (i.e., at local extrema), otherwise it returns the argument with the smallest magnitude. The $\beta$ coefficient is an adjustable parameter in the $1 \leq \beta \leq 2$ range. Using $\beta=1$ results in the minmod limiter as the slope will be determined by one of the first two arguments in equation (10), while $\beta=2$ results in the monotonized central (MC) limiter, which uses the third argument (the central difference slope) as long as it does not violate the TVD property. There are many other possible choices for slope limiters, but for sake of simplicity we only consider this family or limiters here. 
[10] To see the effects of the slope limiter, let us consider a region where some variable and its first derivative are both monotonic, for example

$$
\begin{gathered}
U_{i-1}<U_{i}<U_{i+1}<U_{i+2} \\
U_{i}-U_{i-1}<U_{i+1}-U_{i}<U_{i+2}-U_{i+1}
\end{gathered}
$$

The minmod limiter $(\beta=1)$ will use the smaller (left side in this case) slope to obtain the left and right states

$$
\begin{gathered}
U^{L}=U_{i}+\frac{U_{i}-U_{i-1}}{2}=\frac{3}{2} U_{i}-\frac{1}{2} U_{i-1} \\
U^{R}=U_{i+1}-\frac{U_{i+1}-U_{i}}{2}=\frac{1}{2} U_{i}+\frac{1}{2} U_{i+1}
\end{gathered}
$$

Expanding $U_{i-1}, U_{i}$ and $U_{i+1}$ around $U_{i+1 / 2}$ gives

$$
\begin{aligned}
U^{L} & =U_{i+1 / 2}-\frac{3}{8} \Delta x^{2} \frac{\partial^{2} U}{\partial x^{2}}+O\left(\Delta x^{3}\right) \\
U^{R} & =U_{i+1 / 2}+\frac{1}{8} \Delta x^{2} \frac{\partial^{2} U}{\partial x^{2}}+O\left(\Delta x^{3}\right)
\end{aligned}
$$

and the difference is

$$
U^{R}-U^{L}=\frac{1}{2} \Delta x^{2} \frac{\partial^{2} U}{\partial x^{2}}+O\left(\Delta x^{3}\right)
$$

One can further reduce the dissipative flux with the MC limiter (or any limiter that produces a symmetric formula [see Tóth et al., 2008]). If the solution is smooth enough and the slope limiters do not limit the central slopes then

$$
\begin{aligned}
& U^{L}=U_{i}+\frac{U_{i+1}-U_{i-1}}{4} \\
& U^{R}=U_{i+1}-\frac{U_{i+2}-U_{i}}{4}
\end{aligned}
$$

Expanding $U_{i-1} \ldots U_{i+2}$ around $U_{i+1 / 2}$ gives

$$
U^{R}-U^{L}=\frac{1}{4} \Delta x^{3} \frac{\partial^{3} U}{\partial x^{3}}+O\left(\Delta x^{4}\right)
$$

In general, we can write

$$
U^{R}-U^{L} \propto \Delta x^{n} \frac{\partial^{n} U}{\partial x^{n}}
$$

where $n$ is the local order of the dissipative flux. We note that $n$ is related to, but not the same as, the overall order of accuracy of the whole numerical scheme. In general, the spatial order of the scheme is less than or equal to $n$.

[11] Combining equations (4) and (21), we get

$$
F_{\text {diss }}(U) \approx|\lambda| \Delta x^{n} \frac{\partial^{n} U}{\partial x^{n}}
$$

Our aim is to reduce the dissipation. The last term that expresses the variation of the solution obviously cannot be changed. We can increase $n$ by going to a higher-order scheme, but in unresolved simulations the error is dominated by the cells next to local extrema, where the local order of accuracy drops to $n=1$ for TVD type schemes. We can reduce the grid cell size $\Delta x$ only to the point where the computational cost becomes prohibitive. The only remaining option is to reduce $|\lambda|$. This will reduce the dissipation of the LF and HLL schemes.

\subsection{Boris Correction With Reduced Speed of Light}

[12] One possible way of reducing the wave speed $\lambda$ is using the "Boris correction" with an artificially reduced speed of light. The Boris correction itself [Boris, 1970; Gombosi et al., 2002] means that we are solving the semirelativistic MHD equations instead of the classical MHD equations by keeping the displacement current in the induction equation. Since the displacement current $(1 / c) \partial \mathbf{E} / \partial t$ depends on a time derivative, the steady state solutions of the semirelativistic and classical equations coincide. The timedependent solutions, however, will be different.

[13] In semirelativistic MHD the magnetosonic and Alfvén wave speeds are limited by the speed of light as demanded by the theory of relativity. Near the polar regions of Earth the classical Alfvén speed can approach about a third of the light speed, so the semirelativistic correction has some effect, but not too much. For other planets, like Jupiter or Saturn, the Boris correction is crucial, because the classical Alfvén speed may even exceed the speed of light. For Earth, however, an additional step is required to significantly reduce the numerical diffusion and to allow larger explicit time steps, namely using an artificially reduced speed of light. This will limit $\lambda$ and reduce the numerical diffusion. In addition, this approach allows larger time steps when an explicit time stepping scheme is used, because the numerical stability condition also depends on the largest propagation speed.

[14] The potential drawback of the Boris correction is that the equations are modified by the lowered speed of light. While this has no effect on the steady state solution, the results of time-dependent calculations may not converge to the physically correct solution. Another problem is that the semirelativistic magnetohydrodynamics equations are rather complicated. Simplified versions of the Boris correction exist [Gombosi et al., 2002] and are used in various MHD codes. These are easier to implement but then even the steady state solutions are affected by the lowering of the speed of light. Our MHD code solves for the full set of semirelativistic equations, and all results presented in the paper use this approach.

[15] Certain numerical schemes, like the Roe scheme [Roe, 1981], cannot be combined with the Boris correction (at least in practice), because there are no analytic expressions for the eigenvectors of the semirelativistic MHD equations.

\subsection{Implicit Scheme With Limited Numerical Dissipation}

[16] An alternative new approach to reduce $\lambda$ in equation (22) is to limit the numerical dissipative flux without modifying the underlying physical equations. So instead of using $|\lambda|$ in (4), we use

$$
\lambda^{\prime}=\min (|\lambda|, \Lambda)
$$



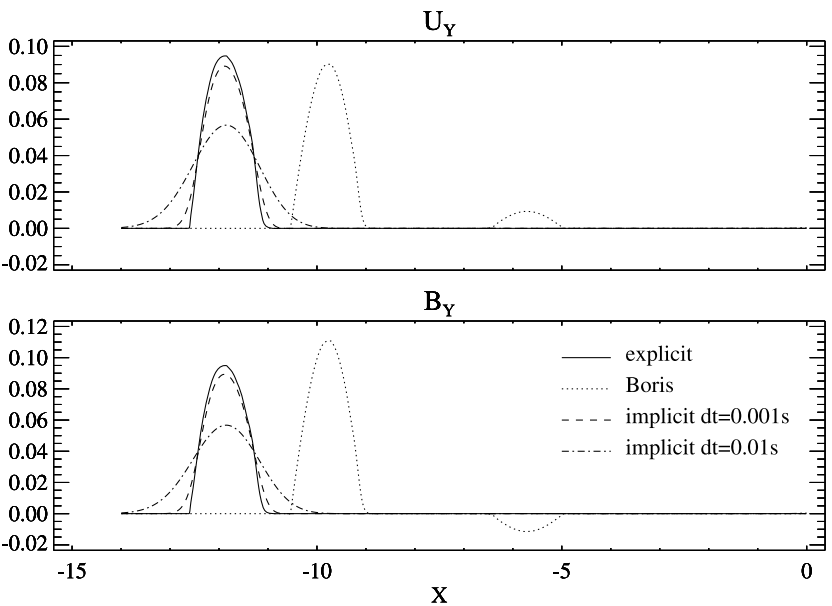

Figure 1. The transverse component of the magnetic field and the velocity at time 3.7 for the Alfvén wave test produced by four different schemes. See text for discussion.

where $\Lambda$ is an adjustable parameter. This means that the wave speeds in the LF, HLL and Roe flux functions in equations (1), (2) and (3) will be replaced as follows

$$
\begin{aligned}
\lambda_{\max } & \rightarrow \min \left(\lambda_{\max }, \Lambda\right) \\
\lambda_{R} & \rightarrow \min \left(\lambda_{R}, \Lambda\right) \\
\lambda_{L} & \rightarrow \max \left(\lambda_{L},-\Lambda\right) \\
\left|\lambda_{k}\right| & \rightarrow \min \left(\left|\lambda_{k}\right|, \Lambda\right)
\end{aligned}
$$

Note that the Roe flux may not benefit much from this modification, since it already uses the proper wave speed for the characteristic waves. Of course, limiting the dissipative flux comes at a price: we have to use an implicit time integration scheme to maintain the stability of the TVD type discretization. Fortunately our BATS-R-US (Block-Adaptive-Tree Solar wind Roe-type Upwind Scheme) code [Powell et al., 1999; Gombosi et al., 2004] has an efficient explicit/implicit time integration scheme [Tóth et al., 2006] that we use in many of our magnetosphere simulations anyways. The other potential drawback is that the reduced diffusion may allow the development of spurious oscillations. Whether this will happen or not in an actual simulation is hard to predict. We know that the Implicit Scheme with Limited Numerical Dissipation, or ISLND for short, works successfully for Hall MHD, where limiting the whistler wave speed in the numerical dissipative flux has been used successfully [Chacón and Knoll, 2003; Tóth et al., 2008]. The advantage of limiting the numerical dissipative flux is that we are still solving the same equations, so the results should converge to the correct physical solution. The ISLND can be easily applied for any numerical flux function that has the form (4).

[17] We adjust $\Lambda$ to significantly reduce the numerical diffusion but we need to make sure that there are no spurious oscillations. For magnetosphere simulations, we found that setting $\Lambda=2000-3000 \mathrm{~km} / \mathrm{s}$ works well, which is the same range as the one typically used for the reduced speed of light $c^{\prime}$ in the Boris scheme.

\section{Numerical Tests}

[18] To clearly demonstrate the properties of the various schemes we have done a couple of simple one dimensional tests. The first test models the propagation of an Alfvén wave, the second involves a static equilibrium of varying magnetic and thermal pressures.

\subsection{Propagation of Alfvén Wave}

[19] The one dimensional grid extends from $-14<x<0$ with periodic boundaries. The initial condition has density $\rho=1$, background magnetic field $B_{x}=3$ aligned with the grid, pressure $p=5$, and the other quantities are zero. We apply a perturbation in the $-1.5<x<0$ range to the transverse velocity $u_{y}=-0.1 \sin (2 \pi x / 3)$ and magnetic field $B_{y}=-0.1 \sin (2 \pi x / 3)$. The analytic solution is an Alfvén wave propagating at $v_{A}=-3$ toward the left boundary. Using $\gamma=5 / 3$ for the polytropic index, the sound speed is $\sqrt{\gamma p / \rho}=\sqrt{25 / 3}=2.89$, so the plasma beta is close to unity.

[20] We use a uniform grid with 1400 grid cells $(\Delta x=$ 0.01 ), so the wave perturbation is very well resolved by 150 cells. All simulations use the LF scheme with Koren's third-order limiter [Koren, 1993] with $\beta=1.5$. Koren's limiter results in third-order accuracy in sufficiently smooth and monotonic regions, but in general, the overall order of accuracy in the L1 norm is limited to second order for all TVD schemes, because the TVD limiters render the accuracy to first order at local extrema which occupy a lower (by one) dimensional subspace of the computational domain [Harten and Osher, 1987]. Nevertheless, according to several numerical tests, Koren's limiter still gives slightly more accurate results than the $\mathrm{MC}$ limiter (10). We also note that in this test the HLL and Roe schemes provide (essentially) identical results with the LF scheme, because the wave propagates at the largest speed, and the $u_{x}$ velocity component is zero.

[21] We are comparing the explicit unmodified scheme, the explicit Boris scheme with a reduced speed of light $c^{\prime}=$ 4.2, and the implicit scheme with two different time steps. Using ISLND with $\Lambda>3$ makes no difference in this case, because the wave speeds are all less than or equal to 3 . More surprisingly, the tests show that using $\Lambda<3$ does not make any difference either, which suggests that for the implicit scheme the numerical error is completely dominated by the time discretization error and the spatial discretization error is negligible. The unmodified explicit scheme takes time steps with $\Delta t \approx 0.0026$ (and 0.0027 for Boris scheme) based on the stability condition. The implicit scheme is run with $\Delta t=$ 0.01 and $\Delta t=0.001$ to show the effect of discretization errors due to the time step.

[22] Figure 1 shows the results at $t=3.7$ when the center of the wave should be at $x=-0.75-3 t=-11.85$. The unmodified explicit scheme produces the most accurate solution. The Boris scheme produces an Alfvén wave that has too large amplitude in the magnetic field, and also propagates slower than the classical Alfvén speed: the center 
B

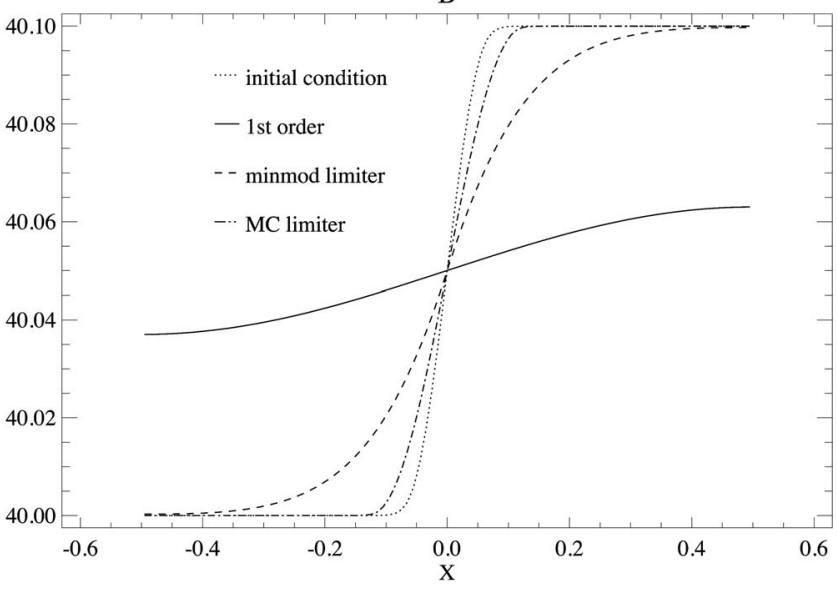

Figure 2. Results of the numerical diffusion test. The transverse magnetic field is shown at the initial time $t=$ 0.2 (dotted line) and at $t=1$ for the various limiters. See main text for discussion.

is at $x \approx-9.8$. In addition there is a second wave with smaller amplitude propagating in the opposite direction (wrapping around the periodic boundaries) at $x \approx-5.7$. These results make perfect sense, since the semirelativistic Alfvén speed [Gombosi et al., 2002] with the reduced speed of light is $v_{A}^{\prime}=v_{A} / \sqrt{1+v_{A}^{2} / c^{\prime 2}} \approx 2.44$. In addition, the initial condition is the exact solution of the left propagating Alfvén wave for the classical, but not for the semirelativistic MHD equations. The amplitude ratio of the transverse velocity and magnetic field perturbations is different in the semirelativistic case, so the initial condition corresponds to a linear combination of a left and a right propagating wave.

[23] The implicit scheme with $\Delta t=0.001$ gives results that are close to the explicit solution, although slightly more diffusive. With larger time steps the diffusion is much more pronounced. This is also expected, since implicit schemes can handle propagating waves accurately only if the waves do not cross more than about a third of a grid cell per time step [Tóth et al., 2006], which corresponds to $\Delta t=0.001$ in this case. For larger time steps the diffusive error becomes quite substantial, although the wave speed and the amplitude ratio remain correct.

\subsection{Static Equilibrium}

[24] The one-dimensional domain extends to $-0.5<x<$ 0.5 and the grid contains 100 cells only. The initial condition is constructed in two stages. First we start from density $\rho=1$, velocity $\mathbf{u}=0$, parallel magnetic field component $B_{x}=$ 0 , and jumps in the transverse field and the thermal pressure: $B_{y}=40, p=4.1$ for $x<0$ and $B_{y}=40.1, p=0.095$ for $x>0$. The sum of the thermal and magnetic pressures $p+B^{2} / 2=$ 804.1 is constant, so this is an equilibrium solution for the ideal MHD equations (also for the semirelativistic equations). In fact, it is also an equilibrium solution for the Roe scheme. For the LF and HLL schemes, on the other hand, numerical diffusion will smooth out the initial discontinuity. To make the comparison of the various schemes more meaningful, we first run the LF scheme (with Koren's limiter and $\beta=1.5$ ) using explicit time stepping up to $t=$
0.2. By this time the initial discontinuity spreads over about a dozen cells. This state at $t=0.2$ is what we regard as the initial condition, and we continue the simulation with the various schemes from here.

[25] First we compare the effect of the order of accuracy. Figure 2 shows the solution at time $t=1$ for the firstorder scheme, the second-order scheme with minmod limiter $(\beta=1)$ and the third-order scheme with the MC limiter $(\beta=$ 1.5). The first-order LF (or HLL) scheme diffuses the solution very rapidly. By $t=5$ the magnetic field is completely flat. The solution obtained with the minmod limiter is less diffused, and the MC (or Koren's) limiter performs even better.

[26] Next let us try to further improve the best result (so far) obtained with the MC limiter. We apply the Boris correction with $c^{\prime}=4.2$, or the ISLND scheme with $\Lambda=4.2$. For sake of comparison we also run the implicit scheme without limiting the diffusion. Since the classical fast magnetosonic speed is about 40.1 everywhere, both the Boris correction and ISLND reduce the maximum wave speed by about a factor of 9.5. The time step of the explicit scheme is $\Delta t \approx 2 \times 10^{-4}$ due to the stability constraints. With the Boris correction, the time step increases to $\Delta t \approx$ $1.9 \times 10^{-3}$ due to the reduction of the fast speed. The implicit scheme can use even larger time steps, and we choose $\Delta t=0.01$ that is 50 times larger than the explicit time step. In this test, the larger time step actually improves the accuracy. We employ Koren's limiter with $\beta=1.5$ in all these runs.

[27] Figure 3 shows the initial condition at $t=0.2$ as a dotted line, while the other lines show the results obtained by the various schemes at the final time $t=5$. The explicit scheme (solid line) is the same as the best scheme (MC limiter) in Figure 2, but here the results are shown at a later time and the $x$ range has been limited to show more detail. The results of the Boris and ISLND schemes are much more accurate (less diffusive) than the unmodified scheme, and these two solutions essentially overlap. This confirms that the semirelativistic and classical MHD equations produce the same solution for steady state problems. It also shows that the Boris and ISLND schemes are equally efficient in

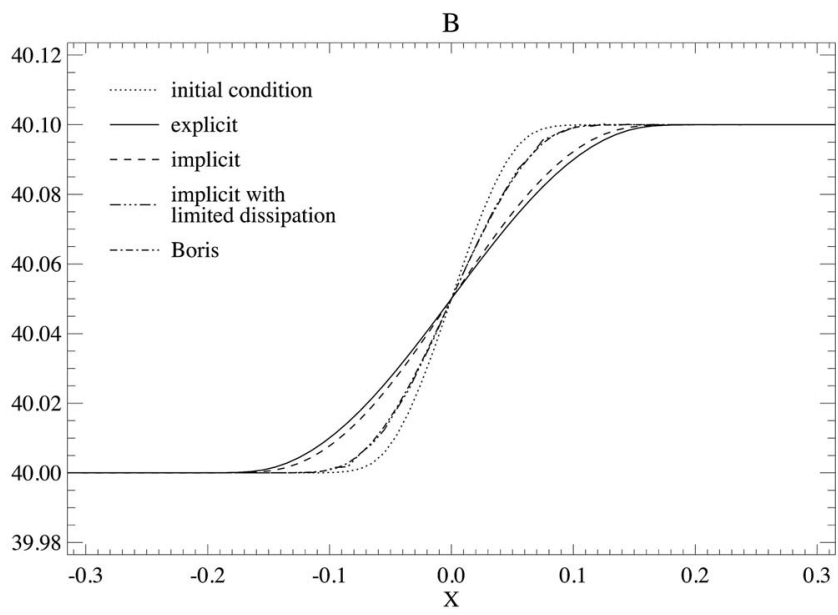

Figure 3. Results of the numerical diffusion test. The transverse magnetic field is shown at the initial time $t=$ 0.2 (dotted line) and at $t=5$ for the various schemes. See text for discussion. 

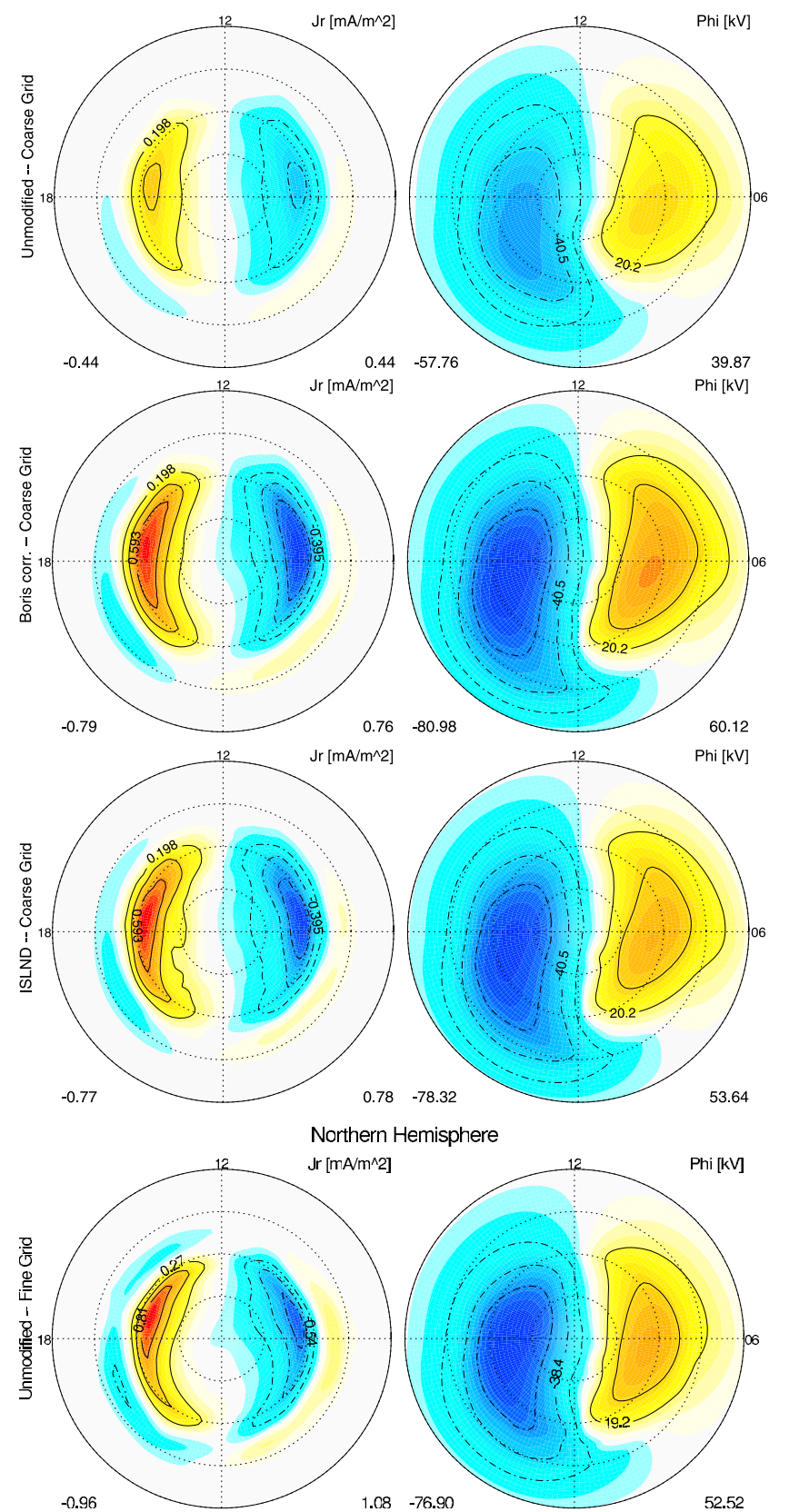

Figure 4. (left) Radial component of the field-aligned currents and (right) the resulting electric potential at 03:00 UT 4 May 1998 obtained with the unmodified artificial wind scheme on the coarser grid, combined with Boris correction with $c^{\prime}=3000 \mathrm{~km} / \mathrm{s}$, combined with limited dissipation with $\Lambda=2000 \mathrm{~km} / \mathrm{s}$, and the unmodified scheme on the higherresolution grid. The center of the plot is the magnetic north pole, the circles indicate magnetic latitudes separated by $10^{\circ}$. The minimum and maximum values are indicated below each plot.

reducing the numerical dissipation. The dashed line shows the result of the unmodified implicit scheme, which is similar but slightly more accurate than the explicit solution. This demonstrates that for steady state (and slowly varying) problems, the implicit time stepping is not more diffusive than the explicit time stepping.

\section{Magnetic Storm Simulations}

[28] We use the Space Weather Modeling Framework (SWMF) [Tóth et al., 2005] to couple the GM, IE and IM models. The global magnetosphere is modeled by the BATSR-US [Powell et al., 1999; Gombosi et al., 2004] code. The ionosphere electrodynamics model is the Ridley Ionosphere Model (RIM), a 2D height integrated potential solver [Ridley et al., 2004]. The inner magnetosphere model is the Rice Convection Model (RCM) [Wolf et al., 1982; Toffoletto et al., 2003].

[29] BATS-R-US provides field-aligned currents to RIM, while RIM returns the electric potential to BATS-R-US, which is used to set the tangential velocities at the inner boundary of BATS-R-US. RIM also sends the electric potential to RCM, while BATS-R-US calculates the magnetic field line volumes and integrates density and pressure along the closed field lines and sends this information to $\mathrm{RCM}$. In return RCM calculates the total pressure from the particle distribution, and sends it to BATS-R-US, and BATS-R-US "nudges" the MHD pressure toward the RCM pressure. Under the right conditions, the increased pressure in the closed field line region leads to field line stretching and the formation of a ring current. Although RCM modifies the MHD solution to some extent, the numerical errors of the MHD scheme play an important role in determining the overall result.
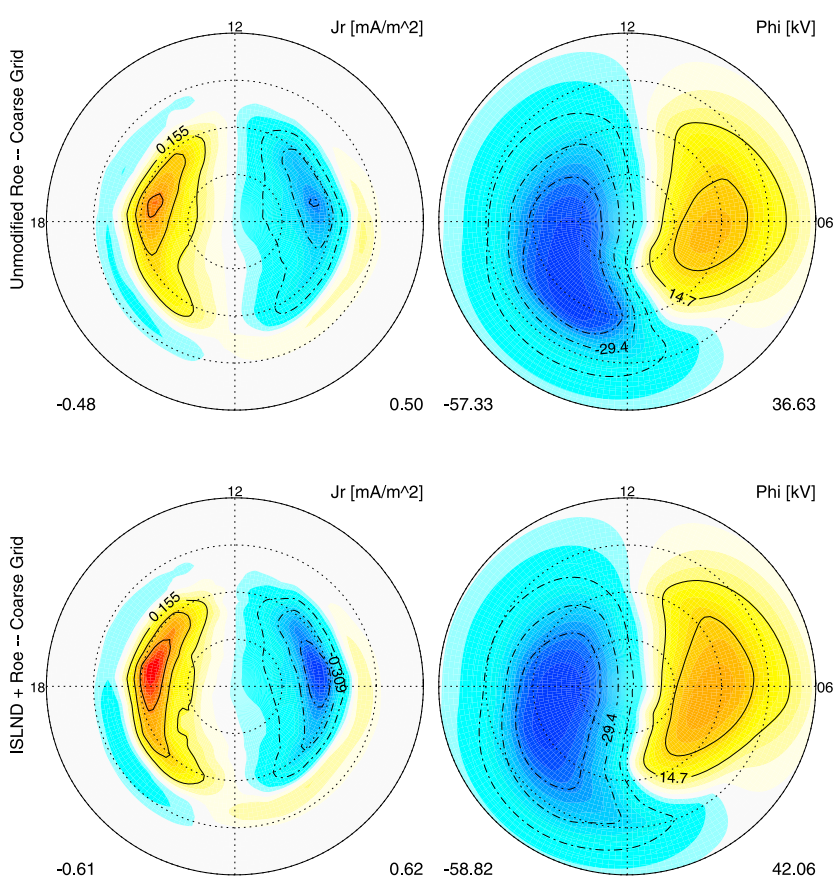

Figure 5. Same as Figure 4 but using the Roe scheme instead of the artificial wind scheme. (left) Radial component of the field-aligned currents and (right) the resulting electric potential (top) with the unmodified Roe scheme and (bottom) with the Roe scheme using limited diffusion with $\Lambda=3000 \mathrm{~km} / \mathrm{s}$. 


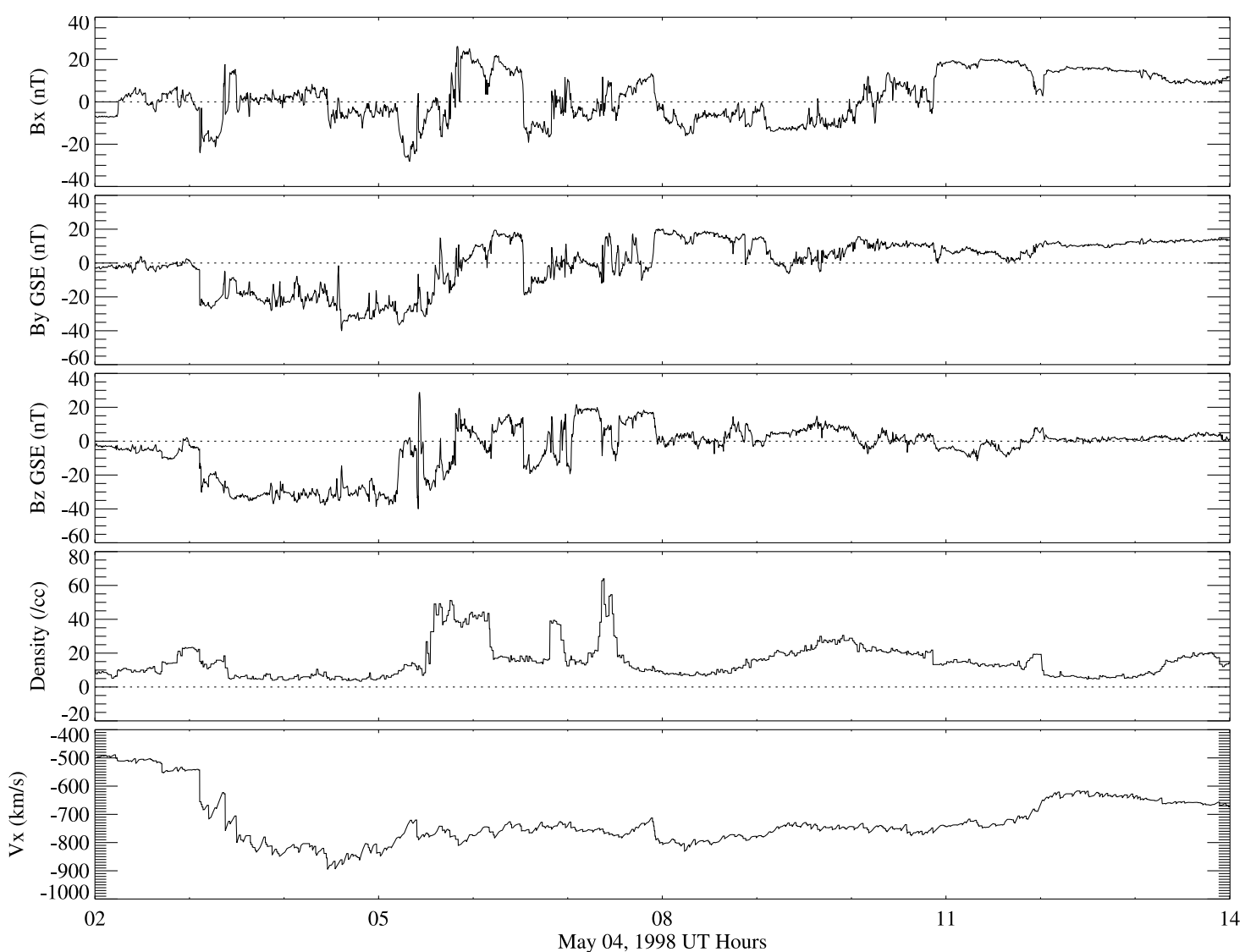

Figure 6. Solar wind condition for the 4 May 1998 storm.

[30] In this study we use the BATS-R-US code with an explicit/implicit scheme [Tóth et al., 2006] with 5 s time steps that may get somewhat reduced by the dynamic time step control scheme. We use the artificial wind (AW) scheme by Sokolov et al. [2002] (which is a variant of the HLL scheme) with Koren's third-order limiter [Koren, 1993] and $\beta=1.2$ unless otherwise noted.

[31] We use a block-adaptive Cartesian mesh (note that BATS-R-US can also use non-Cartesian geometries, including spherical grids). The computational domain extends from $32 \mathrm{R}_{e}$ (Earth radii) upstream to $224 \mathrm{R}_{e}$ downstream of the planet, and $128 \mathrm{R}_{e}$ to the sides. The inner boundary is a sphere of radius $2.5 \mathrm{R}_{e}$ centered on the Earth. The grid resolution varies from $1 / 4 R_{e}$ near the inner boundary to $8 \mathrm{R}_{e}$ near the outer edges for the "low" -resolution grid, and we increase the resolution to $1 / 8 R_{e}$ near the Earth for the "high" -resolution mesh. The low- and high-resolution grids contain about 220,000 and 1.35 million cells, respectively. At the inner boundary the number density is set to $28 / \mathrm{cc}$, the velocity is determined by IE, and the radial component of the magnetic field is fixed to the dipole value. The pressure and the tangential components of the magnetic field are allowed to "float" freely. We set the solar wind conditions upstream of the planet using measurements by the ACE satellite that are propagated to the inflow boundary with the solar wind speed. The other outer boundaries are "float" or "outflow".

[32] The ionosphere model uses a $91 \times 181$ uniform latitudelongitude grid. The star light and the polar cap Pedersen conductances, originating from stellar UV radiation and very light polar rain poleward of the auroral oval, are set to 1 and $0.25 \mathrm{ohm}^{-1}$, respectively. The F10.7 flux index depends on the magnetic storm being modeled, we used 123 sfu for the 4 May 1998 storm and 245 sfu for the 31 March 2001 storm. The IE model is coupled with GM every $5 \mathrm{~s}$. The field-aligned currents are calculated at $3 \mathrm{R}_{e}$ radial distance in GM. The inner magnetosphere model RCM uses the default spatial and energy grids with 5 second time steps. $\mathrm{RCM}$ is coupled with the other two models every $10 \mathrm{~s}$ of simulation time.

[33] We will compare the simulation with the cross polar cap potential (CPCP), and the Dst index calculated with the Assimilative Mapping of Ionospheric Electrodynamics (AMIE) model [Richmond and Kamide, 1988; Ridley and Kihn, 2004], and with in situ satellite measurements of the magnetic field. The AMIE Dst uses all available magnetometers to calculate the average perturbation of the $B_{z}$ component on the surface of the Earth, and it uses a high temporal resolution. In contrast, the Kyoto Dst index is based on 4 magnetometers and is averaged over 1 hour periods. Both of these indexes are approximations of the global magnetic perturbation parallel to the dipole axis at the center of the Earth. In practice, the AMIE Dst is easier to compare with the simulation results, because it provides a more accurate estimate of the global magnetic perturbation, and it has a higher temporal resolution. 


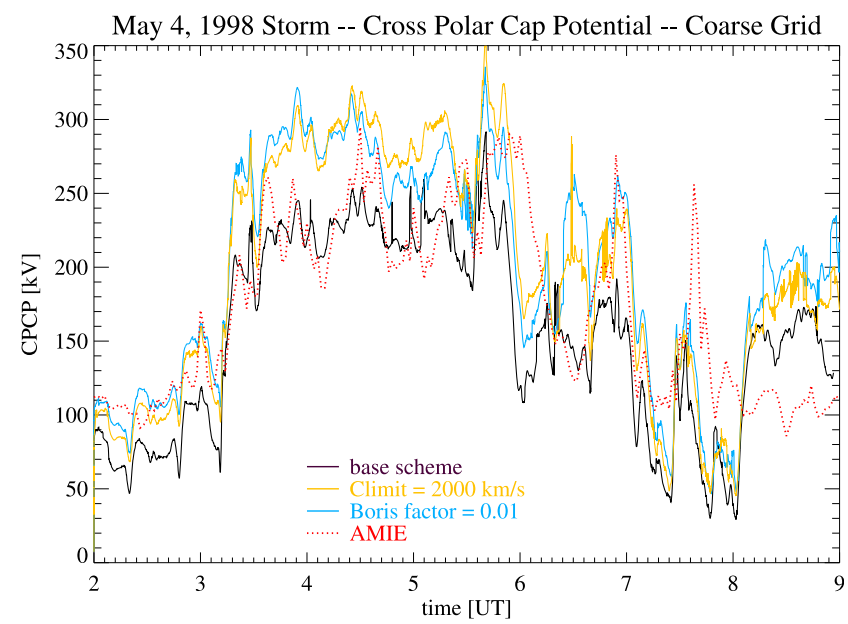

Figure 7. Northern cross polar cap potential obtained by three different schemes on the coarser grid (solid lines) compared with AMIE results (red dotted line).

[34] The simulated CPCP is calculated by the ionospheric model as the difference between the largest and smallest potential on the northern hemisphere. The simulated Dst index is calculated by the global magnetosphere model as the average value of the $B_{z}$ component of the magnetic field taken over a spherical surface at $R=3.0 \mathrm{R}_{e}$. The simulated Dst index neglects the effect of currents inside $3 \mathrm{R}_{e}$, but it still provides a reasonable approximation for the global magnetic perturbation (see Yu and Ridley [2008] for a detailed discussion). Finally, the in situ magnetic field values are obtained by interpolating the magnetic field from the global magnetosphere grid to the position of the satellite as it moves along its trajectory.

\subsection{Comparison of Field-Aligned Currents and Electric Potentials}

[35] To examine the effect of reduced numerical diffusion on the FAC and the electric potential, we compare snapshots from the ionospheric electrodynamics model at 03:00 UT in the 4 May 1998 storm simulation.

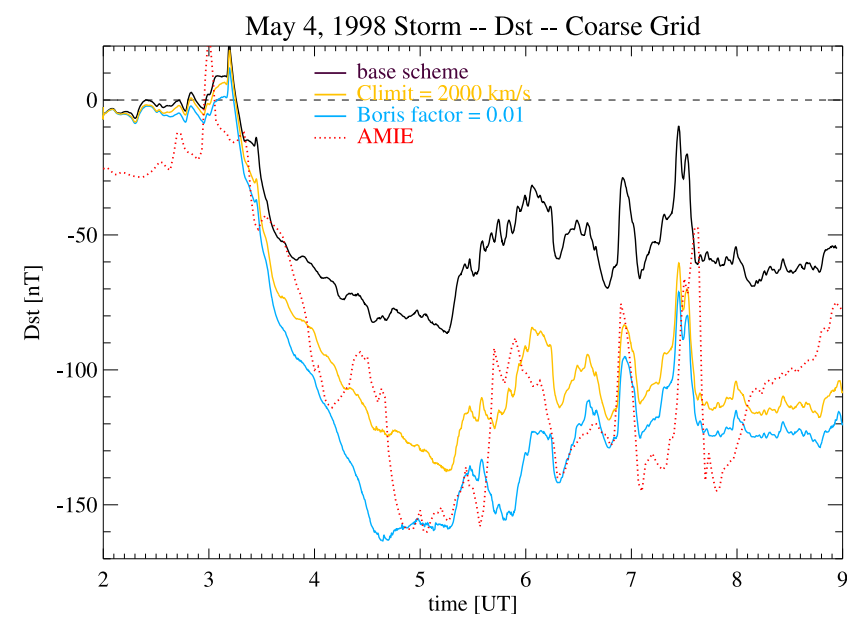

Figure 8. Dst indexes obtained by three different schemes on the coarser grid (solid lines) compared with AMIE results (red dotted line).

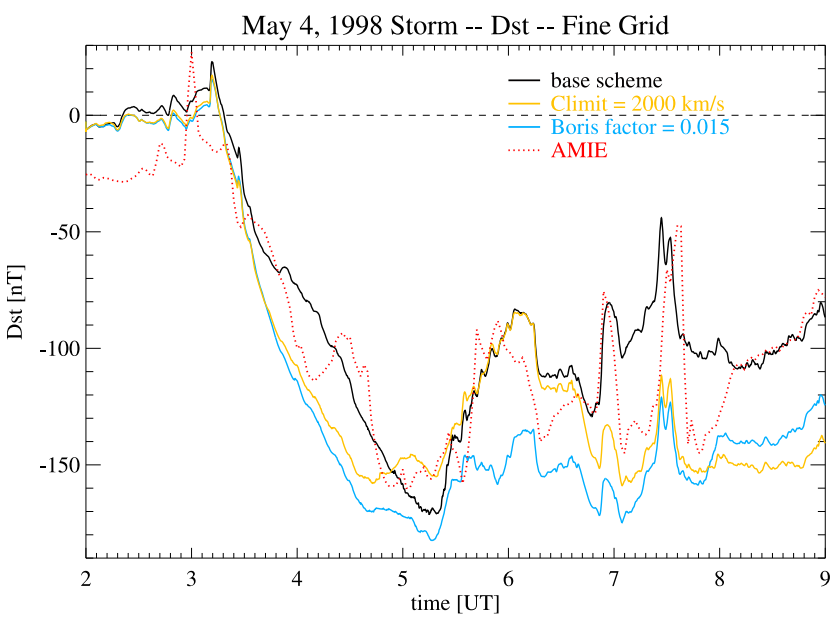

Figure 9. Dst indexes obtained by three different schemes on the finer grid (solid lines) compared with AMIE results (red dotted line).

[36] Figure 4 compares the unmodified artificial wind scheme on the coarser and finer grids, as well as the AW scheme combined with Boris correction with $c^{\prime}=3000 \mathrm{~km} / \mathrm{s}$ and the limited dissipation scheme with $\Lambda=2000 \mathrm{~km} / \mathrm{s}$ on the coarser grid. Note that the ISLND scheme applies a sharp limit on $|\lambda|$ at $\Lambda$, while the Boris correction already reduces $|\lambda|$ before it would reach the lowered speed of light $c^{\prime}$. This means that $\Lambda$ should be set to be somewhat smaller than $c^{\prime}$ to achieve comparable reduction in the numerical diffusion.

[37] We find that both the Boris and ISLND schemes result in sharper and stronger field-aligned currents and more extended and stronger electric potential than the unmodified scheme on the same grid. The reduced diffusion results agree quite well with the results obtained from the higher grid resolution run.

[38] Figure 5 shows results obtained with the Roe scheme. The Roe scheme can only be combined with the ISLND scheme, and it is not a priori obvious if there should be any improvement. We had to use $\Lambda=3000 \mathrm{~km} / \mathrm{s}$ for the Roe scheme to avoid numerical problems. Interestingly, the trends are similar to those found with the AW scheme, there is some improvement in the FAC, but less significant. The differences between the Roe and AW solutions are further explored by Ridley et al. [2010]. In the following simulations we use the AW scheme.

\subsection{The 4 May 1998 Storm}

[39] Figure 6 shows the solar wind parameters for the 4 May 1998 storm. We start the simulation by obtaining an approximate steady state solution with the 02:00 UT solar wind conditions. Then we switch to time-accurate mode and run the coupled model for $12 \mathrm{~h}$ of simulation time.

[40] We ran the SWMF six times using the base scheme, the Boris correction, and the ISLND method on both the coarser and finer grids. For the ISLND we set the maximum wave speed in the dissipative flux to $\Lambda=2000 \mathrm{~km} / \mathrm{s}$. For the Boris correction we used a reduced speed of light $c^{\prime}=$ $3000 \mathrm{~km} / \mathrm{s}$ on the coarse grid, and $c^{\prime}=4500 \mathrm{~km} / \mathrm{s}$ on the finer grid (the code failed with negative pressure for lower $c^{\prime}$ values on the fine grid). 
May 4, 1998 Storm -- Magnetic Field with GOES 8 -- Coarse Grid
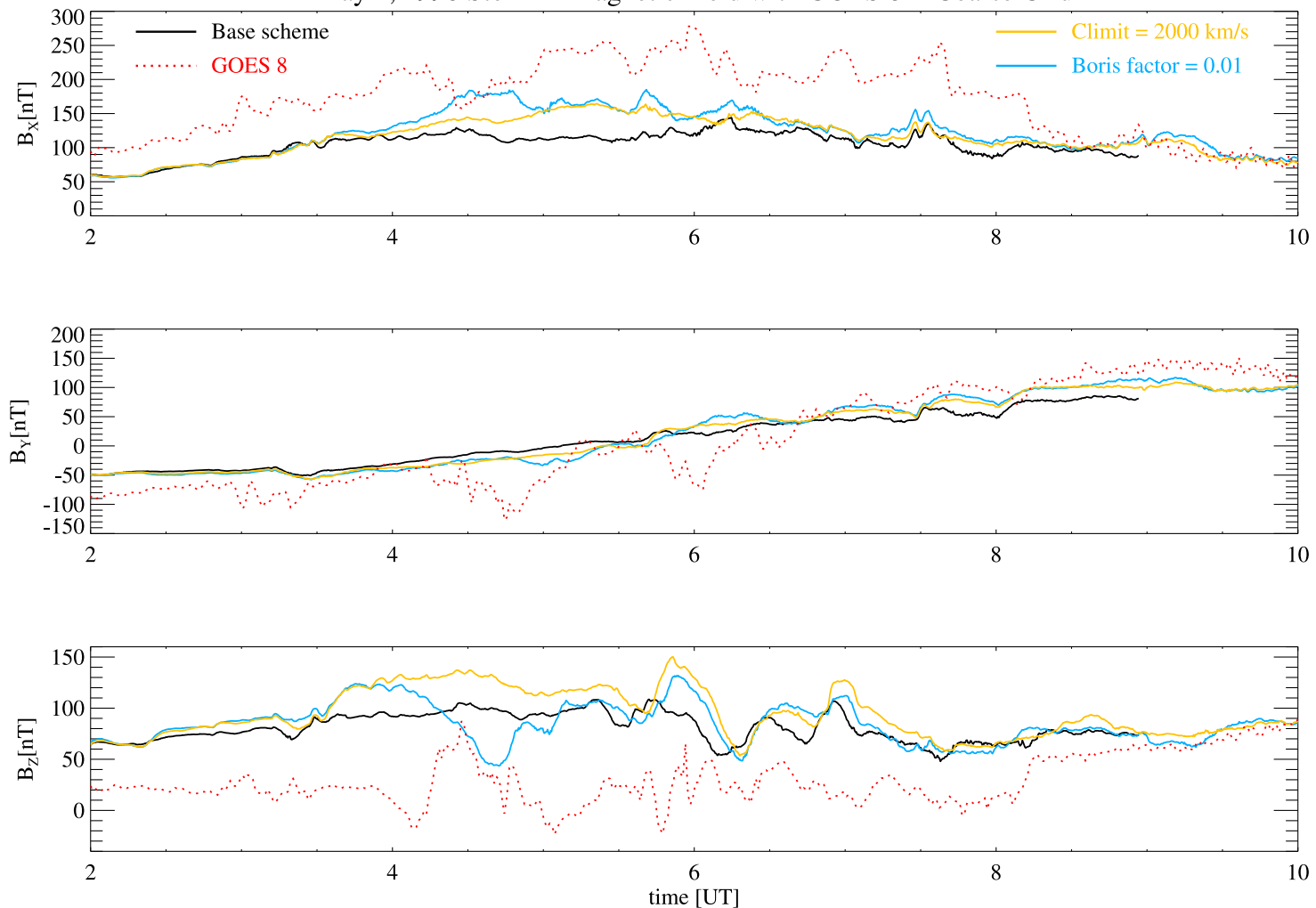

Figure 10. Magnetic field components obtained by three different schemes on the coarser grid (solid lines) compared with GOES-8 date (red dotted line) along the satellite trajectory.
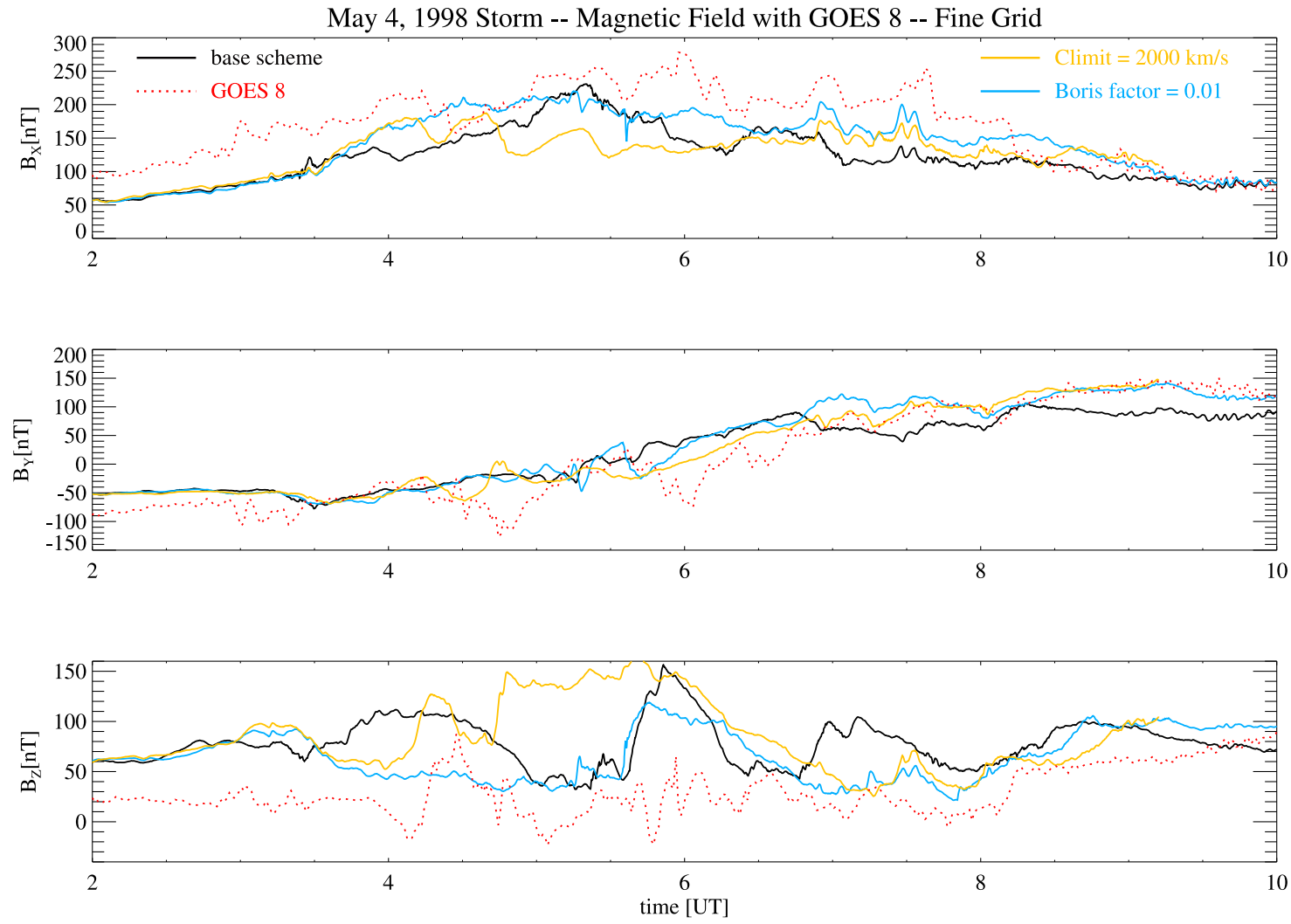

Figure 11. Magnetic field components obtained by three different schemes on the finer grid (solid lines) compared with GOES-8 date (red dotted line) along the satellite trajectory. 


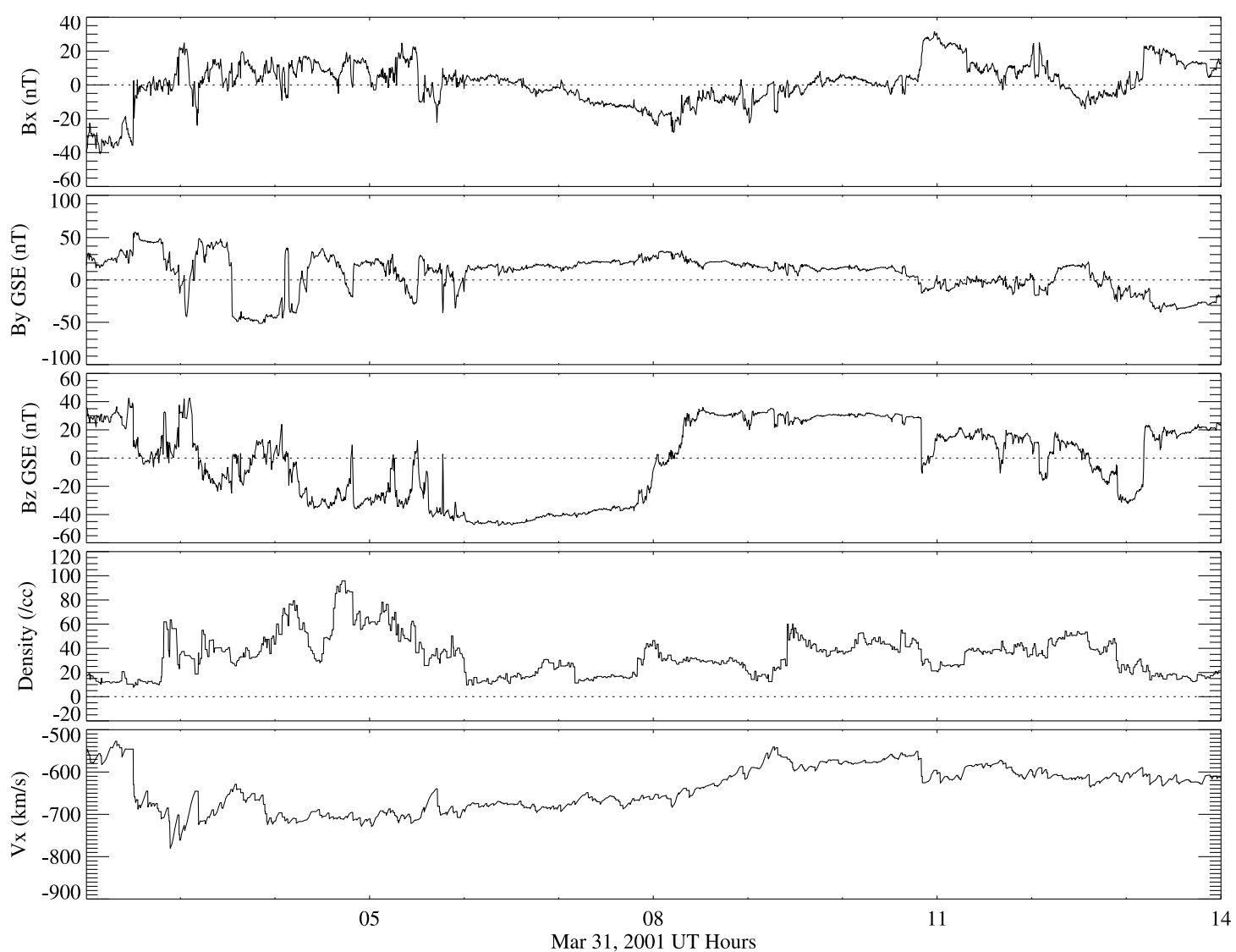

Figure 12. Solar wind condition for the 31 March 2001 storm.

[41] We plot the simulated and assimilated northern cross polar cap potentials (CPCP) for the coarse grid in Figure 7. The CPCP is clearly higher when the numerical diffusion is reduced by either the Boris correction or by the ISLND. Interestingly, the assimilated values agree somewhat better with the base scheme. This is probably due to the tuning of the empirical relations used in the RIM code.

[42] Figure 8 compares the Dst indexes for the coarse grid. The base scheme produces a Dst index that is about factor of 2 or more smaller (in absolute value) than the results obtained by the Boris correction and limited diffusion schemes. The latter schemes produce Dst indexes that agree reasonably well with the AMIE data.

[43] Figure 9 compares the Dst indexes for the finer grid, and we can see that all three schemes are quite close to the assimilated data. In fact the base scheme agrees remarkably well with the AMIE results on the finer grid. The Boris scheme produces a somewhat too negative Dst for the whole storm, while the limited diffusion scheme is too negative in the "recovery" phase mostly.

[44] Finally, Figures 10 and 11 compare the simulated magnetic field components with in situ measurements by the GOES-8 satellite on the coarse and fine grids, respectively. Overall the simulated field is more dipolar $\left(B_{x}\right.$ is smaller and $B_{z}$ is larger) than the GOES- 8 data. For the coarse grid, the reduced numerical diffusion improves the agreement somewhat, but on the finer grid the three methods produce similar results, and none of those agree well with the data. It is quite likely that the reason is missing physics in the model.
Adding ionospheric outflow seems to improve the agreement substantially [Glocer et al., 2009a, 2009b], probably due to the increased density that results in a more enhanced ring current, and more stretching of the magnetic field.

\subsection{The 31 March 2001 Storm}

[45] Figure 12 shows the solar wind parameters for the 31 March 2001 storm. We start the simulation at 00:00 UT and finish at 16:00 UT.

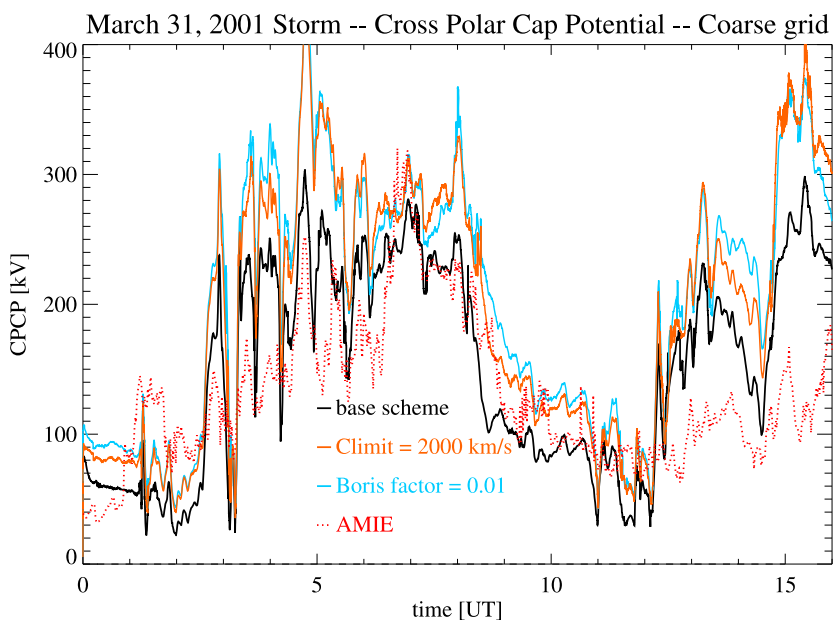

Figure 13. Northern cross polar cap potential obtained by three different schemes on the coarser grid (solid lines) compared with AMIE results (red dotted line). 


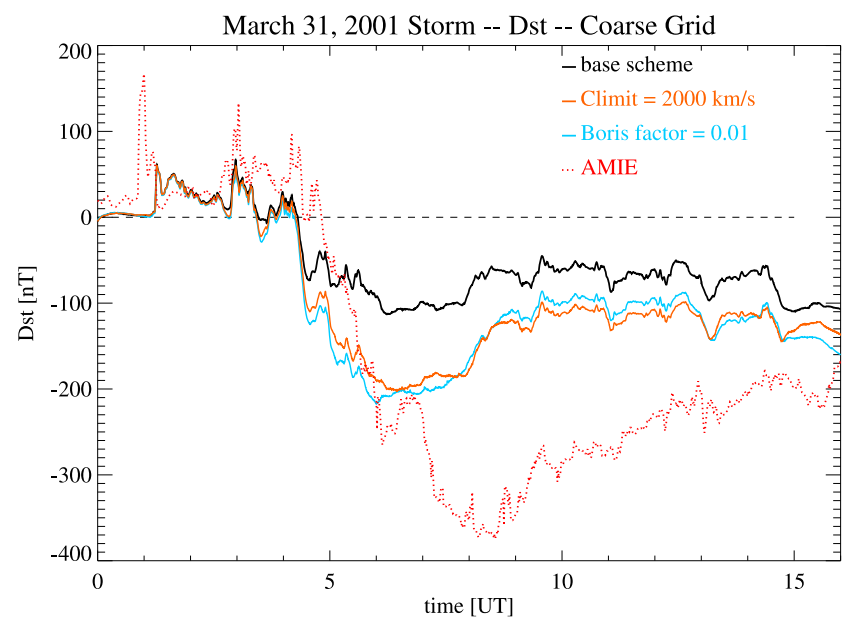

Figure 14. Dst indexes obtained by three different schemes on the coarser grid (solid lines) compared with AMIE results (red dotted line).

[46] We ran the SWMF six times using the base scheme, the Boris correction, and the ISLND on both the coarser and finer grids. For the Boris correction we used a reduced speed of light $c^{\prime}=3000 \mathrm{~km} / \mathrm{s}$. For ISLND we set $\Lambda=2000 \mathrm{~km} / \mathrm{s}$ on the coarse grid, and $\Lambda=3000 \mathrm{~km} / \mathrm{s}$ on the finer grid (the code failed for lower $\Lambda$ values on the finer grid).

[47] Figures 13 and 14 compare the simulated and assimilated northern CPCP and Dst indexes, respectively, for the coarser grid. The CPCP is higher and the Dst is more negative when the numerical diffusion is reduced. The agreement with the assimilated data is not very good: the simulated CPCP is too high for most of the time period, while the Dst is not negative enough after 07:00 UT.

[48] Figures 15 and 16 show the same indexes for the finer grid. The simulated CPCP gets higher and the differences between the 3 schemes get smaller when compared with the coarser grid. Unfortunately this makes the deviation larger with respect to the AMIE CPCP results. The simulated Dst index gets more negative and differences are smaller among the 3 schemes on the finer grid. The agree-

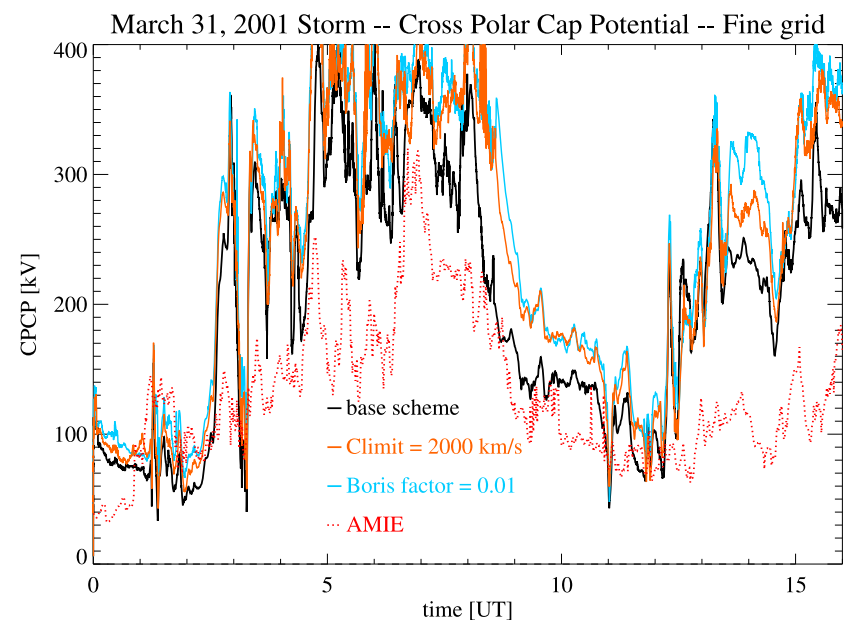

Figure 15. Northern cross polar cap potential obtained by three different schemes on the finer grid (solid lines) compared with AMIE results (red dotted line).

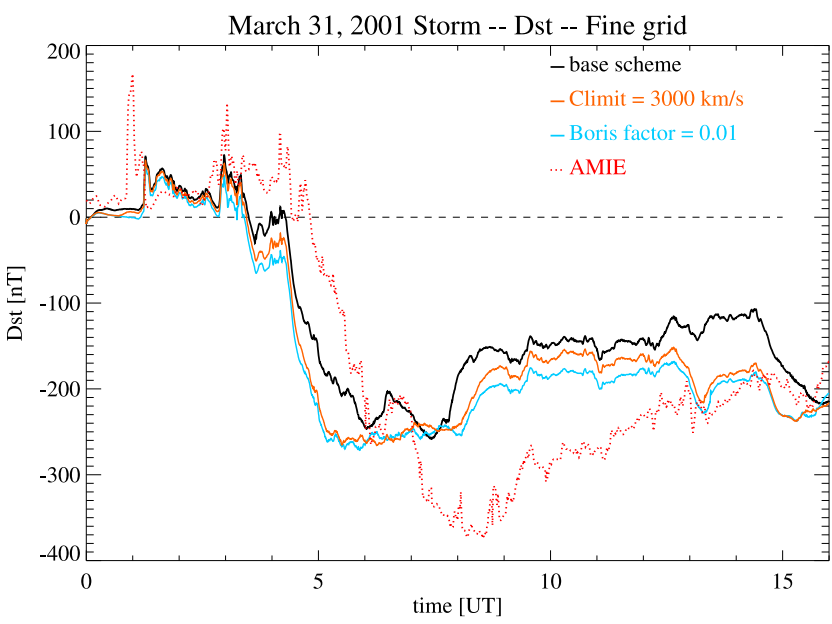

Figure 16. Dst indexes obtained by three different schemes on the finer grid (solid lines) compared with AMIE results (red dotted line).

ment with AMIE calculated Dst is somewhat better in the period after 7:00 UT, on the other hand the Dst drops too steeply from 2:00 UT to 5:00 UT.

[49] Overall the trends are the same as in the May 1998 storm simulations. The reduced numerical diffusion makes a large difference for the coarser grid, and somewhat less difference on the finer grid. The Dst index is more negative with the reduced numerical diffusion and/or for the finer grid. Including ionospheric outflow can improve the agreement with the measured Dst index [Glocer et al., 2009a].

\section{Conclusions}

[50] We have examined how the numerical diffusion and its effects can be reduced in MHD simulations. We compared various numerical flux functions and different slope limiters. The Roe scheme is generally less diffusive than the HLL scheme, which in turn is less diffusive than the LaxFriedrichs scheme. In some problems, however, the Roe scheme cannot be used, either because it is not robust enough for the application of interest, or because the eigenvectors of the equations are too complicated. Using sharp limiters (e.g., MC or Koren's limiter) increases the order of accuracy and reduces the diffusion. Again, robustness issues may not allow the use of the sharpest possible limiter. The optimal choice of the flux function and slope limiter is problem dependent.

[51] We compared the "classical" Boris correction method with a new approach ISLND that limits the dissipative numerical flux but does not change the equations. We presented simple tests demonstrating that the Boris scheme may converge to an incorrect solution in time dependent problems. The implicit scheme converges to the correct solution, as long as the time step is kept small enough. For large time steps there are significant diffusive and/or dispersive numerical errors for fast moving waves. In steady state (or slowly varying) problems both the Boris correction and the ISLND scheme give accurate results. In this case one can use large time steps for the implicit scheme without losing accuracy.

[52] In the magnetospheric simulations, we found that both the Boris correction and the ISLND methods reduce the 
numerical diffusion in a similar fashion compared to the unmodified implicit scheme. This is most clear in the Dst index that gets much more negative compared to the base scheme. As we increase the grid resolution, the differences between the three schemes become smaller. This is a sign of getting closer to a grid converged solution with respect to the global indexes. The GOES satellite comparison shows that the point-wise values are still rather far from the observations, even for the higher grid resolution. This is probably due to some missing physics. We found that including ionospheric outflow can greatly improve the agreement with the GOES data [Glocer et al., 2009a, 2009b].

[53] Overall we find that both the Boris and ISLND methods are effective in reducing the numerical diffusion and they produce comparable results in magnetospheric simulations. This is not a priori obvious, since the Boris scheme changes the time-accurate equations. Both numerical diffusion reduction approaches are much less expensive than increasing the grid resolution. We found that the limited diffusion scheme is more expensive than the Boris scheme, because it requires more iterations from the implicit scheme to solve the large linear system of equations. This is probably caused by the increased stiffness of the matrix when the dissipative flux is reduced.

[54] The ISLND scheme can serve as a relatively inexpensive (compared to a full grid convergence study) check for the correctness of the results obtained with the Boris correction. It can also be used for equations and schemes for which the Boris correction is not applicable.

[55] Acknowledgments. This research was supported by the AISR NASA grant NNX07AV80G.

[56] Philippa Browning thanks the reviewers for their assistance in evaluating this paper.

\section{References}

Boris, J. P. (1970), A physically motivated solution of the Alfvén problem, Tech. Rep. NRL Memo. Rep. 2167, Nav. Res. Lab, Washington, D. C.

Chacón, L., and D. A. Knoll (2003), A 2D high- $\beta$ Hall MHD implicit nonlinear solver, J. Comput. Phys., 188, 573-592, doi:10.1016/S0021-9991 (03)00193-1.

De Zeeuw, D., S. Sazykin, R. Wolf, T. Gombosi, A. Ridley, and G. Tóth (2004), Coupling of a global MHD code and an inner magnetosphere model: Initial results, J. Geophys. Res., 109, A12219, doi:10.1029/ 2003JA010366.

Glocer, A., G. Tóth, T. Gombosi, and D. Welling (2009a), Modeling ionospheric outflows and their impact on the magnetosphere, initial results, J. Geophys. Res., 114, A05216, doi:10.1029/2009JA014053.

Glocer, A., G. Tóth, Y. Ma, T. Gombosi, J. -C. Zhang, and L. M. Kistler (2009b), Multifluid Block-Adaptive-Tree Solar wind Roe-type Upwind Scheme: Magnetospheric composition and dynamics during geomagnetic storms: Initial results, J. Geophys. Res., 114, A12203, doi:10.1029/ 2009JA014418

Gombosi, T. I., G. Tóth, D. L. De Zeeuw, K. C. Hansen, K. Kabin, and K. G. Powell (2002), Semi-relativistic magnetohydrodynamics and physicsbased convergence acceleration, J. Comput. Phys., 177, 176-205, doi:10.1006/jcph.2002.7009.

Gombosi, T. I., et al. (2004), Solution-adaptive magnetohydrodynamics for space plasmas: Sun-to-Earth simulations, Comput. Sci. Eng., 6(2), 14-35, doi:10.1109/MCISE.2004.1267603.

Harten, A. (1983), High resolution schemes for hyperbolic conservation laws, J. Comput. Phys., 49, 357-393, doi:10.1016/0021-9991(83) 90136-5.

Harten, A., and S. Osher (1987), Uniformly high-order accurate nonoscillatory schemes. I, SIAM J. Numer. Anal., 24, 279-309, doi:10.1137/ 0724022 .
Harten, A., P. D. Lax, and B. van Leer (1983), On upstream differencing and Godunov-type schemes for hyperbolic conservation laws, SIAM Rev., 25(1), 35-61, doi:10.1137/1025002.

Koren, B. (1993), A robust upwind discretisation method for advection, diffusion and source terms, in Numerical Methods for Advection-Diffusion Problems, edited by C. Vreugdenhil and B. Koren, pp. 117-138, Vieweg, Brunswick, Germany.

Lyon, J., J. Fedder, and C. Mobarry (2004), The Lyon-Fedder-Mobarry (LFM) global MHD magnetospheric simulation code, J. Atmos. Sol. Terr. Phys., 66, 1333-1350, doi:10.1016/j.jastp.2004.03.020.

Palmroth, M., T. I. Pulkkinen, P. Janhunen, and C.-C. Wu (2003), Stormtime energy transfer in global MHD simulation, J. Geophys. Res., 108(A1), 1048, doi:10.1029/2002JA009446.

Powell, K., P. Roe, T. Linde, T. Gombosi, and D. L. De Zeeuw (1999), A solution-adaptive upwind scheme for ideal magnetohydrodynamics, J. Comput. Phys., 154, 284-309, doi:10.1006/jcph.1999.6299.

Raeder, J., et al. (2001), Global simulation of the Geospace Environment Modeling substorm challenge event, J. Geophys. Res., 106, 381-395, doi:10.1029/2000JA000605.

Richmond, A., and Y. Kamide (1988), Mapping electrodynamic features of the high-latitude ionosphere from localized observations: Technique, J. Geophys. Res., 93, 5741-5759, doi:10.1029/JA093iA06p05741.

Ridley, A. J., and E. A. Kihn (2004), Polar cap index comparisons with AMIE cross polar cap potential, electric field, and polar cap area, Geophys. Res. Lett., 31, L07801, doi:10.1029/2003GL019113.

Ridley, A. J., T. I. Gombosi, and D. L. Dezeeuw (2004), Ionospheric control of the magnetosphere: conductance, Ann. Geophys., 22, 567-584, doi:10.5194/angeo-22-567-2004.

Ridley, A. J., D. L. D. Zeeuw, T. I. Gombosi, K. C. Hansen, I. V. Sokolov, G. Toth, and D. T. Welling (2010), Numerical considerations in simulating the global magnetosphere, Ann. Geophys., 28, 1589-1614, doi:10.5194/angeo-28-1589-2010.

Roe, P. L. (1981), Approximate Riemann solvers, parameter vectors, and difference schemes, J. Comput. Phys., 43, 357-372, doi:10.1016/00219991(81)90128-5.

Rusanov, V. V. (1970), On difference schemes of third order accuracy for nonlinear hyperbolic systems, J. Comput. Phys., 5, 507-516, doi:10.1016/0021-9991(70)90077-X.

Sokolov, I., E. V. Timofeev, J. Sakai, and K. Takayama (2002), Artificial wind-A new framework to construct simple and efficient upwind shock-capturing schemes, J. Comput. Phys., 181, 354-393, doi:10.1006/ jcph.2002.7130.

Tanaka, T. (1995), Generation mechanisms for magnetosphere-ionosphere current systems deduced from a three-dimensional MHD simulation of the solar wind-magnetosphere-ionosphere coupling process, J. Geophys. Res., 100(A7), 12,057-12,074, doi:10.1029/95JA00419.

Toffoletto, F., S. Sazykin, R. Spiro, and R. Wolf (2003), Inner magnetospheric modeling with the Rice Convection Model, Space Sci. Rev. 107, 175-196, doi:10.1023/A:1025532008047.

Toffoletto, F., S. Sazykin, R. Spiro, R. Wolf, and J. Lyon (2004), RCM meets LFM: Initial results of one-way coupling, J. Atmos. Sol. Terr. Phys., 66, 1361-1370, doi:10.1016/j.jastp.2004.03.022.

Tóth, G., et al. (2005), Space Weather Modeling Framework: A new tool for the space science community, J. Geophys. Res., 110, A12226, doi:10.1029/2005JA011126.

Tóth, G., D. L. De Zeeuw, T. I. Gombosi, and K. G. Powell (2006), A parallel explicit/implicit time stepping scheme on block-adaptive grids, J. Comput. Phys., 217, 722-758, doi:10.1016/j.jcp.2006.01.029.

Tóth, G., Y. J. Ma, and T. I. Gombosi (2008), Hall magnetohydrodynamics on block adaptive grids, J. Comput. Phys., 227, 6967-6984, doi:10.1016/ j.jcp.2008.04.010

van Leer, B. (1979), Towards the ultimate conservative difference scheme. V. A second-order sequel to Godunov's method, J. Comput. Phys., 32, 101-136, doi:10.1016/0021-9991(79)90145-1.

Wolf, R. A., M. Harel, R. W. Spiro, G. Voigt, P. H. Reiff, and C. K. Chen (1982), Computer simulation of inner magnetospheric dynamics for the magnetic storm of July 29, 1977, J. Geophys. Res., 87, 5949-5962, doi:10.1029/JA087iA08p05949.

Yu, Y., and A. Ridley (2008), Validation of the Space Weather Modeling Framework using ground-based magnetometers, Space Weather, 6, S05002, doi:10.1029/2007SW000345.

T. I. Gombosi, X. Meng, A. J. Ridley, and G. Tóth, Department of Atmospheric Oceanic and Space Science, University of Michigan, 2455 Hayward, Ann Arbor, MI 48109, USA. (gtoth@umich.edu) 